\title{
Ion-Collision Emission Excitation Cross Sections for Xenon Electric Thruster Plasmas
}

\author{
Jason D. Sommerville* and Lyon B. King $\ddagger$ \\ Michigan Technological University, Houghton, Michigan 49931 \\ and \\ Yu-Hui Chiu走 and Rainer A. Dressler $\underline{\S}$ \\ U.S. Air Force Research Laboratory, Hanscom Air Force Base, Massachusetts 01731
}

\section{DOI: $\underline{10.2514 / 1.33657}$}

\begin{abstract}
Energetic xenon ion collisions with neutral xenon atoms play an important role in electric thruster plasma radiation at low electron temperatures. The respective emission excitation cross sections are necessary for the derivation of plasma parameters from the observed radiance. We present apparent emission excitation cross sections for near-infrared $5 p^{5} 6 p$ to $5 p^{5} 6$ s transitions of neutral xenon impacted by singly and doubly charged xenon ions. The cross sections were measured over a laboratory energy-per-charge-number range of 100 to $900 \mathrm{eV}$, a range that covers typical Hall effect thruster discharge voltages. The cross sections are derived from ion beam luminescence spectra produced at single-collision conditions and at pressures for which radiation trapping effects were shown to be negligible. The $\mathrm{Xe}^{+}$cross sections are significantly higher than those of $\mathrm{Xe}^{2+}$ and increase with energy throughout the investigated range. The $\mathrm{Xe}^{2+}$ cross sections plateau at approximately $600 \mathrm{eV}$. The cross sections are incorporated in a collisional-radiative model. The calculations of near-infrared spectra demonstrate that the sensitivity of the model diagnostic with respect to electron temperature increases with ion energy.
\end{abstract}

\section{Nomenclature}

$\begin{array}{ll}B(\lambda)=\text { uncorrected background spectrum } \\ E & =\text { kinetic energy } \\ e & =\text { electronic charge } \\ f(E) & =\text { energy distribution function } \\ G & =\text { geometric correction factor } \\ I & =\text { ion beam current } \\ J_{\lambda} & =\text { radiation intensity in photons per second for transition } \\ & \\ k_{\lambda} & =\text { at } \lambda \\ M & =\text { metastable enhancement factor } \\ m & =\text { atom or ion mass } \\ n_{0} & =\text { neutral density } \\ n_{e} & =\text { electron density } \\ P & =\text { target gas pressure } \\ q & =\text { species charge number } \\ R(\lambda) & =\text { uncorrected spectrum } \lambda \\ S & =\text { sensitivity of emission line intensity ratio to electron } \\ & \text { temperature. } \\ s(\lambda) & =\text { detector spectral sensitivity } \\ T_{e} & =\text { electron temperature } \\ t & =\text { exposure time } \\ \alpha_{1} & =\text { singly charged ion fraction } \\ \Delta \lambda & =\text { peak integration width } \\ \lambda & =\text { wavelength }\end{array}$

Received 23 July 2007; revision received 12 February 2008; accepted for publication 2 March 2008. This material is declared a work of the U.S. Government and is not subject to copyright protection in the United States. Copies of this paper may be made for personal or internal use, on condition that the copier pay the $\$ 10.00$ per-copy fee to the Copyright Clearance Center, Inc., 222 Rosewood Drive, Danvers, MA 01923; include the code 0748-4658/ $08 \$ 10.00$ in correspondence with the CCC.

${ }^{*}$ Graduate Research Assistant, Mechanical Engineering and Engineering Mechanics Department, 815 R. L. Smith Building, 1400 Townsend Drive. Student Member AIAA.

${ }^{\dagger}$ Associate Professor, Mechanical Engineering and Engineering Mechanics Department, 815 R. L. Smith Building, 1400 Townsend Drive. Member AIAA.

${ }^{\ddagger}$ Staff Scientist, Space Vehicles Directorate, 29 Randolf Road. Member AIAA.

${ }^{\S}$ Currently Principle Scientist, Spectral Sciences, Inc., 4 Fourth Avenue, Burlington, MA 01803-3304. Member AIAA.

$$
\begin{array}{ll}
\xi_{\lambda} & =\begin{array}{l}
\text { unscaled emission excitation cross section at } \\
\text { wavelength } \lambda
\end{array} \\
\sigma_{\lambda} & =\text { emission excitation cross section at wavelength } \lambda
\end{array}
$$

\section{Introduction}

$\mathbf{X}$ ENON is used as a propellent in electric spacecraft thrusters, including Hall effect thrusters (HETs) and gridded ion thrusters. These thrusters use electric and magnetic fields to create a plasma and expel the ions at high velocity to generate thrust [1]. The development and testing of these thrusters requires diagnostic techniques that can provide information on plasma properties such as species densities and electron temperature. Optical emission spectroscopy [2] is an attractive diagnostic because it is entirely passive and noninvasive; therefore, it guarantees that the plasma is not disturbed in the process of taking the measurement. Furthermore, it can be applied to remote observations or to examine regions of thruster plasma that are too harsh for electrostatic probes. Observed spectra of absolute line intensities can contain sufficient information to derive electron energy distributions and electron number densities [2-6]. Researchers studying HETs have applied optical emission spectroscopy to investigate general emissive properties [7-9] , plasma fluctuations [10-12], transient operation [13], and thruster body erosion $[14,15]$.

The extraction of quantitative plasma parameters from observed radiance relies on an emission model that describes the intensity of radiation from two or more transition lines as a function of plasma parameters such as electron temperature and species densities. Central to most of these models are emission cross section data. The cross sections are necessary to determine the emission excitation rate coefficient:

$$
k_{\lambda}=\int_{0}^{\infty} \sqrt{\frac{2 E}{m}} \sigma_{\lambda}(E) f(E) \mathrm{d} E
$$

where $f(E)$ is normalized. As Eq. (1) implies, cross sections are collision-energy-dependent. HET plasma electrons typically have a broad energy distribution; therefore, a model requires cross sections over a wide range of electron energies. In contrast, ion-energy distributions in HETs are narrow; typical full widths at halfmaximum are about $15 \%$ of the acceleration voltage and are centered at energies about $10 \%$ below the corresponding acceleration voltage 
$[16,17]$. Therefore, a single set of ion cross sections is sufficient to model an HET operating at a fixed discharge voltage, provided these cross sections change slowly over the range of ion energies present in the HET beam.

The model must incorporate cross sections for all processes that give rise to significant emission on lines within a spectral region of interest. A comprehensive list of xenon emission excitation processes that affect plasma radiation include

$$
\begin{aligned}
\mathrm{e}^{-}+\mathrm{Xe} & \rightarrow \mathrm{Xe}^{*}+\mathrm{e}^{-} \\
& \rightarrow \mathrm{Xe}^{n+*}+(n+1) \mathrm{e}^{-} \\
\mathrm{e}^{-}+\mathrm{Xe}^{+} & \rightarrow \mathrm{Xe}^{n+*}+n \mathrm{e}^{-} \\
\mathrm{e}^{-}+\mathrm{Xe}^{m} & \longrightarrow \mathrm{Xe}^{*}+\mathrm{e}^{-} \\
& \rightarrow \mathrm{Xe}^{n+*}+(n+1) \mathrm{e}^{-} \\
\mathrm{Xe}^{+}+\mathrm{Xe} & \longrightarrow \mathrm{Xe}^{+*}+\mathrm{Xe}^{*} \\
\mathrm{Xe}^{2+}+\mathrm{Xe} & \longrightarrow \mathrm{Xe}^{2+*}+\mathrm{Xe}^{*} \\
& \longrightarrow \mathrm{Xe}^{+*}+\mathrm{Xe}^{+*}
\end{aligned}
$$

where an asterisk denotes excited emitting atoms or ions, and $m$ denotes metastable atoms.

Until recently, a lack of accurate emission excitation cross sections for many of these processes limited the utility of optical emission spectroscopy in determining electron temperature and other plasma parameters in xenon-propelled electric thrusters. This problem was circumvented by applying a simple collisional-radiative model such as the coronal equilibrium model $[2,3,18]$. The corona model assumes that all excitation occurs from electron collisions with ground-state neutral atoms; that is, only process 2 is considered. This greatly reduces the need for cross section data. Bouchoule et al. [13], however, demonstrated experimentally that metastables play a fundamental role in the HET plasma radiance spectrum, and therefore processes 5 and 6 cannot be ignored. Meanwhile, Chiu et al. [19] reported cross sections for ion emission excitation (processes 8 and 9) for 300-eV ions, demonstrating that they are large for nearinfrared lines. Karabadzhak et al. [20] developed a more rigorous collisional-radiative model (CRM) for the near-infrared spectrum of xenon thruster plasmas. In addition to electron collisions with ground-state neutrals (process 2), the model also considers excitation from metastable states (process 5) and excitation due to heavyparticle collisions using the cross sections of Chiu et al. [19]. The results of Karabadzhak et al. [20] demonstrated that metastables can enhance emission intensities of particular lines by factors as high as three, depending on the electron temperature. Karabadzhak et al. also showed that ion-neutral collisions are a significant excitation mechanism at low electron temperatures, such as may be encountered in the plume of an HET. To reduce the number of coupled state-to-state transition rate equations necessarily introduced by the added processes, the CRM makes use of apparent emission cross sections. These cross sections include emission contributions from cascade transitions from higher levels into the upper level as well as direct excitation to the upper level from the ground state.

The CRM of Karabadzhak et al. [20] applies to the emission lines associated with the lowest excited states of neutral xenon: specifically, the transitions from the $5 p^{5} 6 p(2 p$ in Paschen notation) upper states to the $5 \mathrm{p}^{5} 6 \mathrm{~s}(1 \mathrm{~s})$ lower states (Table 1). These lines have high intensities and occur in a relatively sparse region of the spectrum, thereby avoiding experimental problems due to line interference, and cross section data for many of the reactions are
Table 1 Xenon emission lines studied

\begin{tabular}{lccc}
\hline \hline Upper state & Branching ratio & Lower state & $\lambda, \mathrm{nm}$ \\
\hline $2 \mathrm{p}_{1}$ & $0.720 \pm 0.059$ & $1 \mathrm{~s}_{2}$ & 788.74 \\
$2 \mathrm{p}_{3}$ & $0.863 \pm 0.050$ & $1 \mathrm{~s}_{2}$ & 834.68 \\
$2 \mathrm{p}_{5}$ & $0.998 \pm 0.000$ & $1 \mathrm{~s}_{4}$ & 828.01 \\
$2 \mathrm{p}_{6}$ & $0.743 \pm 0.041$ & $1 \mathrm{~s}_{5}$ & 823.16 \\
$2 \mathrm{p}_{6}$ & $0.243 \pm 0.039$ & $1 \mathrm{~s}_{4}$ & 895.23 \\
$2 \mathrm{p}_{7}$ & $0.901 \pm 0.020$ & $1 \mathrm{~s}_{4}$ & 916.27 \\
$2 \mathrm{p}_{7}$ & $0.099 \pm 0.019$ & $1 \mathrm{~s}_{5}$ & 840.92 \\
$2 \mathrm{p}_{8}$ & $1.000 \pm 0.000$ & $1 \mathrm{~s}_{5}$ & 881.94 \\
$2 \mathrm{p}_{9}$ & $0.620 \pm 0.029$ & $1 \mathrm{~s}_{4}$ & 992.32 \\
$2 \mathrm{p}_{9}$ & $0.379 \pm 0.046$ & $1 \mathrm{~s}_{5}$ & 904.54 \\
$2 \mathrm{p}_{10}$ & $0.975 \pm 0.032$ & $1 \mathrm{~s}_{5}$ & 979.97 \\
\hline \hline
\end{tabular}

${ }^{\mathrm{a} C a l c u l a t e d ~ f r o m ~ e l e c t r o n-i m p a c t ~ e x c i t a t i o n ~ c r o s s ~ s e c t i o n s ~ g i v e n ~ i n ~[21] . ~}$

available. Because the subject lines involve only emissions from neutral xenon, processes $3,4,6$, and 9 may be ignored. Cross sections for process 2 have recently been measured over a broad range of energies $[19,21]$. The model includes an approximate method based on cascade population of metastable states to account for process 5 .

Before this work, the CRM of Karabadzhak et al. [20] was limited to thrusters with discharge voltages of approximately $300 \mathrm{~V}$. Although $300 \mathrm{~V}$ may be regarded as the most common thruster discharge voltage, HETs operate over a range of voltages from $100 \mathrm{~V}$ [22] to $1000 \mathrm{~V}$ [23-27]. Most gridded ion thrusters operate above $1000 \mathrm{~V}$, but some work has been done on low-power and lowspecific-impulse ion thrusters that used voltages in the 600-900-V range $[28,29]$. Given the demonstrated importance of ion-collisioninduced emission excitation for $300-\mathrm{V}$ discharge voltages and the possibility that the respective emission excitation cross sections depend strongly on energy, measurements of cross sections for processes 7 and 8 over a broad energy range are necessary to apply the diagnostic model to engines with other discharge voltages. To extend the applicability of the Karabadzhak et al. [20] CRM to a wider range of thruster operating points, we measured the apparent emission cross sections for singly and doubly charged ion impact of neutral xenon for the $E / q$ range from 100 to $900 \mathrm{eV}$ for the transitions listed in Table 1 . The new cross sections are incorporated into the CRM of Karabadzhak et al., and the effect of HET discharge voltage on the predicted near-infrared xenon plasma radiation spectrum is discussed.

\section{Experiment}

The measurements were performed on the ion beam luminescence apparatus of the U.S. Air Force Research Laboratory at Hanscom Air Force Base. The high-vacuum apparatus, schematically shown in Fig. 1, was previously described by Chiu et al. [19], who measured cross sections for 300-eV-per-charge ions. In summary, a glowdischarge ion source generates a xenon ion beam. The beam passes through a Wien velocity filter that selects the desired ionization level. The beam is then focused into a collision cell filled with a xenon target gas. After passing through the cell, the beam current is collected by a Faraday cup. To assess the divergence of the ion beam, the beam current to the Faraday cup and the back wall of the cell are monitored with electrometers, and the sum of the currents is used to quantify the beam current. As the beam enters the Faraday cup, it passes through a retarding potential analyzer (RPA), which is used to verify the beam energy before the luminescence experiment is run. The target gas pressure is monitored with a capacitance manometer. Both target pressure and beam current are continuously monitored throughout each experiment and the time-averaged values are used. The face of a fiber optical bundle collects luminescence photons produced in the cell and the fibers guide the photons out of the vacuum chamber to a spectrograph equipped with a charge-coupled device detector.

The spectral sensitivity of the spectrograph was calibrated by comparing the recorded spectrum of a halogen-tungsten lamp with the Planck distribution for a body at the temperature of the lamp, as measured by a pyrometer. The temperature of the lamp was found to 


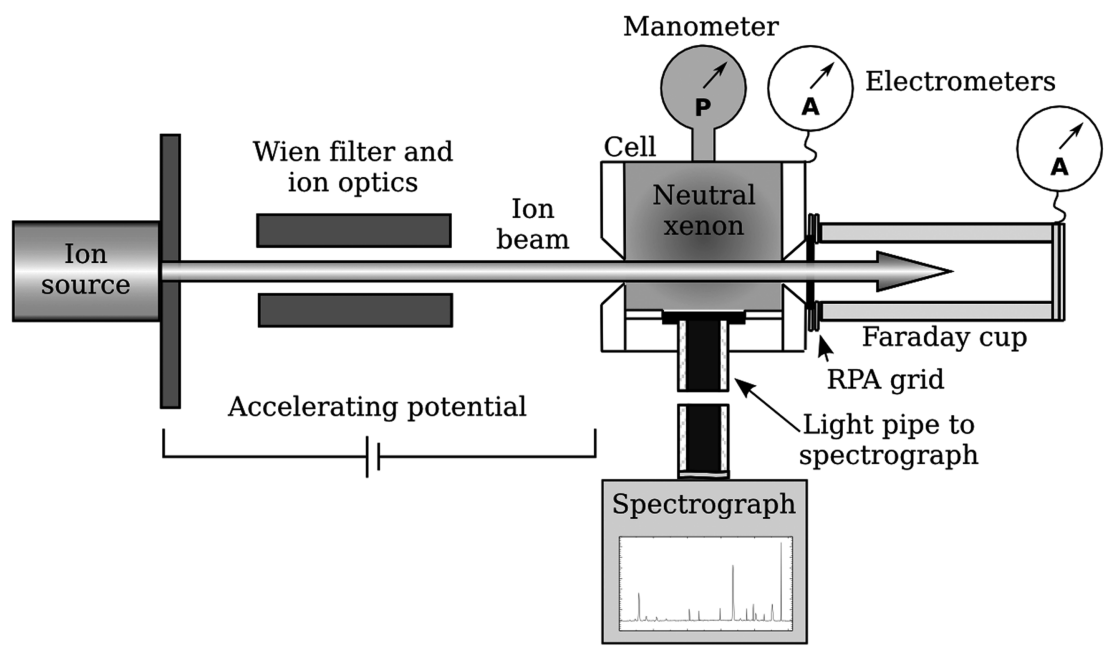

Fig. 1 Schematic representation of the ion excitation cross section experiment.

be $3065 \mathrm{~K}$, close to the manufacturer's specified nominal temperature of $3200 \mathrm{~K}$. The intensity in photons per unit time of a line at wavelength $\lambda$ was calculated by first correcting the raw spectra for background light, the spectral sensitivity of the detection system and the exposure time, and then integrating over a fixed spectral width:

$$
J_{\lambda}=\int_{\lambda-\frac{1}{2} \Delta \lambda}^{\lambda+\frac{1}{2} \Delta \lambda} s\left(\lambda^{\prime}\right) \frac{R\left(\lambda^{\prime}\right)-B\left(\lambda^{\prime}\right)}{t} \mathrm{~d} \lambda^{\prime}
$$

The integration width was chosen to approximate the full width at half-maximum of the spectral peaks. The widths were 0.6 and $1 \mathrm{~nm}$ for experiments run with 50- and $100-\mu$ m entrance slits, respectively.

The cross sections are derived from the spectral measurements of a given transition line according to

$$
\sigma_{\lambda}=\frac{q e J_{\lambda}}{G P I}
$$

All experiments were conducted with a cell pressure of 1.5 mtorr. Chiu et al. [19] showed that radiation trapping effects are insignificant for ion excitation of the studied emission lines between the pressures of 0.1 and 2.0 mtorr. The geometric correction factor $G$ accounts for the effective solid angle of observation and detector efficiency. It was experimentally determined by comparing the $\mathrm{Xe}^{+}+\mathrm{Xe}$ reaction at $300 \mathrm{eV}$ with the results previously obtained at this energy on the same apparatus [19]. We define the unscaled cross section as Eq. (11) with $G=1$ :

$$
\xi_{\lambda}=\frac{q e J_{\lambda}}{P I}
$$

If $\sigma_{i}$ are the values reported by Chiu et al. [19] for different transitions $i$, and $\xi_{i}$ are the respective measured unscaled cross sections, then $G$ is given by the average:

$$
G=\left\langle\frac{\xi_{i}}{\sigma_{i}}\right\rangle
$$

The $G$ factor is used to scale all measurements, yielding the absolute cross sections.

There are two major contributions to error in the experiment. The largest contribution arises from the uncertainty in the $G$ factor, and the estimate for this contribution is given by the standard deviation of the individual ratios averaged in Eq. (13), which yields a 30\% error. The second major contribution is the uncertainty in the spectralsensitivity calibration. We estimate this error by the largest deviation between the presently determined line ratios for lines with common upper states and the average of values derived from previously published data, as shown in Table 2. The data listed under experiment 1 are derived from measurements of electron-impact excitation cross sections by Fons and Lin [21]. Experiment 2 refers to the ratios of Einstein $A$ coefficients listed by Radzig and Smirnov [30]. The theoretical values from Aymar and Coulombe [31] are the ratios of $A$ coefficients that were calculated with a central field model. The largest deviation of the present ratios from the average of these values is $22.8 \%$. We therefore conservatively estimate a $25 \%$ spectral-sensitivity calibration error for all lines. Inspection of the data suggests that the spectral-sensitivity calibration error most strongly affects the 916- and 905-nm transitions. Combining the $G$ factor and spectral-sensitivity contributions, the error on all measurements at $E / q$ of $200 \mathrm{eV}$ and greater is estimated at $40 \%$. For lines on which multiple measurements were conducted, the standard deviations were checked and all were within this $40 \%$ estimate. For the $100-\mathrm{eV}$ measurements, the errors are estimated at $60 \%$, except for the $841-\mathrm{nm}$ line, for which the error is estimated at $85 \%$. Note that this line is not used in the CRM of Karabadzhak et al. [20], because it has the same upper state $\left(2 \mathrm{p}_{7}\right)$ as the $916-\mathrm{nm}$ line, which is significantly more intense. The higher errors at $100 \mathrm{eV}$ are due to smaller signal levels combined with less stable beam operation and high divergence at this low energy.

\section{Results}

Figure 2 shows 2p-line emission excitation cross sections for $\mathrm{Xe}^{+}+\mathrm{Xe}^{-}$collisions as a function of ion energy (laboratory frame). In the case of the $2 p_{6}, 2 p_{7}$, and $2 p_{9}$ upper levels, two lines are

Table 2 Comparison of line ratios

\begin{tabular}{llllcccc}
\hline \hline Upper state & \multicolumn{1}{c}{$\lambda_{1} / \lambda_{2}$} & \multicolumn{1}{c}{ Exp. 1 } & \multicolumn{1}{c}{ Exp. 2 } & Theory & Average & Present & $\%$ deviation \\
\hline $2 \mathrm{p}_{6}$ & $823 \mathrm{~nm} / 895 \mathrm{~nm}$ & $3.06 \pm 0.653$ & $2.87 \pm 0.406$ & 2.36 & 2.76 & $2.65 \pm 0.211$ \\
$2 \mathrm{p}_{7}$ & $916 \mathrm{~nm} / 841 \mathrm{~nm}$ & $9.10 \pm 1.95$ & $10.8 \pm 1.53$ & 14.0 & 11.3 & $13.0 \pm 4.71$ \\
$2 \mathrm{p}_{9}$ & $992 \mathrm{~nm} / 905 \mathrm{~nm}$ & $1.64 \pm 0.350$ & $1.60 \pm 0.226$ & 1.81 & 1.68 & $1.30 \pm 0.178$ & -22.8 \\
\hline \hline
\end{tabular}

${ }^{a}$ Calculated from electron-impact excitation cross sections given in [21].

${ }^{\mathrm{b} C a l c u l a t e d}$ from Einstein $A$ coefficients given in [30].

${ }^{c}$ Calculated from Einstein $A$ coefficients given in [31] . 

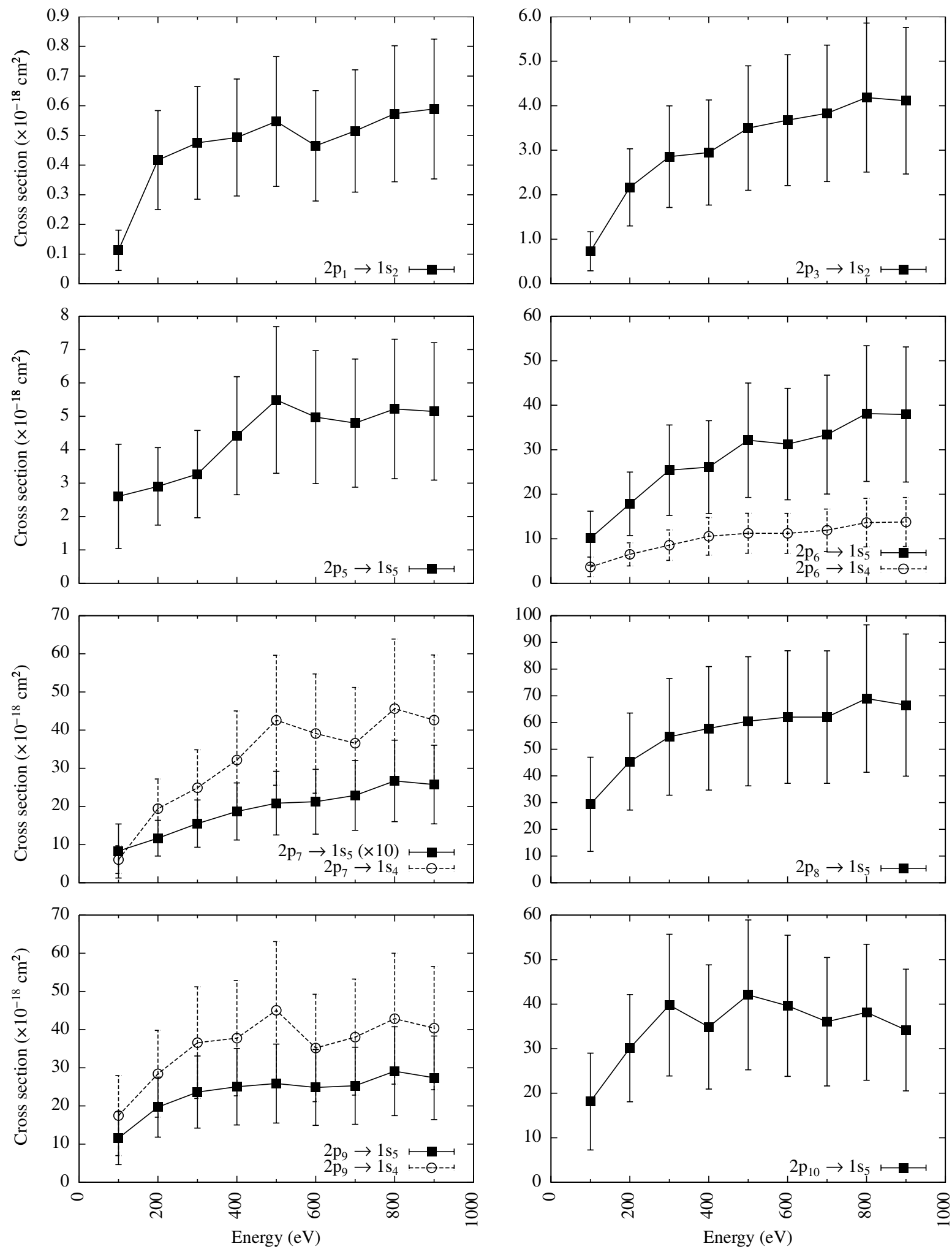

Fig. $2 \mathrm{Xe}^{+}+\mathrm{Xe}$ emission excitation cross sections as a function of ion energy.

produced in the near-infrared spectrum, and the emission excitation cross sections for both lines are shown. Except for the 980-nm line originating in the $2 \mathrm{p}_{10}$ level, all emission excitation cross sections are shown to increase with ion energy throughout the measured ionenergy range. In the case of the 980-nm line, the cross section becomes constant with energy above $300 \mathrm{eV}$. The cross section values are listed in Table 3. The largest cross section of $69 \times$ $10^{-18} \mathrm{~cm}^{2}$ is observed for the $2 \mathrm{p}_{8}$ line at an energy of $800 \mathrm{eV}$, whereas the maximum electron-induced emission excitation cross section is approximately $38 \times 10^{-18} \mathrm{~cm}^{2}$ at $15 \mathrm{eV}$ for the same line [21].

Figure 3 shows $2 \mathrm{p}$-line emission excitation cross sections for $\mathrm{Xe}^{2+}+\mathrm{Xe}$ collisions as a function of ion energy. Again, transitions that share a common upper state are plotted together. All cross sections appear to plateau at $600 \mathrm{eV}$. The cross section values are listed in Table 4 . The $789-\mathrm{nm} \mathrm{Xe} \mathrm{Xe}^{2+}$ cross section at $200 \mathrm{eV}$ was 
Table $3 \mathrm{Xe}^{+}+\mathrm{Xe}$ emission excitation cross sections $\left(\mathbf{1 0}^{-18} \mathrm{~cm}^{2}\right)^{\mathrm{a}}$

\begin{tabular}{|c|c|c|c|c|c|c|c|c|c|c|c|}
\hline Energy, eV & $789 \mathrm{~nm}$ & $823 \mathrm{~nm}$ & $828 \mathrm{~nm}$ & $835 \mathrm{~nm}$ & $841 \mathrm{~nm}$ & $882 \mathrm{~nm}$ & $895 \mathrm{~nm}$ & $905 \mathrm{~nm}$ & $916 \mathrm{~nm}$ & $980 \mathrm{~nm}$ & $992 \mathrm{~nm}$ \\
\hline 100 & $0.112^{\underline{b}}$ & $10.1^{\mathrm{b}}$ & $2.60 \underline{\underline{b}}$ & $0.729 \underline{b}$ & $0.832 \mathrm{c}$ & $29.3^{\mathrm{b}}$ & $3.67 \underline{\mathrm{b}}$ & $11.5^{\mathrm{b}}$ & $6.05^{\mathrm{b}}$ & $18.1^{\mathrm{b}}$ & $17.4^{\mathrm{b}}$ \\
\hline 200 & 0.416 & 17.8 & 2.90 & 2.16 & 1.16 & 45.3 & 6.49 & 19.6 & 19.4 & 30.1 & 28.4 \\
\hline 300 & 0.475 & 25.4 & 3.27 & 2.85 & 1.55 & 54.6 & 8.55 & 23.6 & 24.8 & 39.7 & 36.5 \\
\hline 400 & 0.493 & 26.1 & 4.42 & 2.94 & 1.86 & 57.8 & 10.5 & 25.0 & 32.1 & 34.8 & 37.7 \\
\hline 500 & 0.547 & 32.1 & 5.49 & 3.49 & 2.08 & 60.4 & 11.2 & 25.8 & 42.5 & 42.0 & 45.0 \\
\hline 600 & 0.464 & 31.2 & 4.97 & 3.67 & 2.12 & 62.0 & 11.2 & 24.8 & 39.0 & 39.6 & 35.1 \\
\hline 700 & 0.515 & 33.3 & 4.79 & 3.82 & 2.28 & 62.0 & 11.9 & 25.2 & 36.5 & 36.0 & 38.0 \\
\hline 800 & 0.573 & 38.1 & 5.22 & 4.18 & 2.66 & 68.9 & 13.6 & 29.1 & 45.6 & 38.1 & 42.8 \\
\hline 900 & 0.589 & 37.9 & 5.14 & 4.11 & 2.57 & 66.5 & 13.7 & 27.3 & 42.6 & 34.1 & 40.3 \\
\hline
\end{tabular}

${ }^{a}$ All measurements have a $40 \%$ uncertainty unless otherwise marked

${ }^{\mathrm{b}} 60 \%$ uncertainty

c85\% uncertainty

unobtainable due to the low signal-to-noise ratio for that peak. It is denoted by a dash in the table. Except for the 789-nm line, all cross sections are smaller than their $\mathrm{Xe}^{+}$-induced counterparts by roughly an order of magnitude. The largest cross section is approximately $13 \times 10^{-18} \mathrm{~cm}^{2}$, observed for the $882-\mathrm{nm}$ line at $600 \mathrm{eV}$.

\section{Discussion}

The presently determined energy dependencies of emission excitation cross sections in $\mathrm{Xe}^{+}+\mathrm{Xe}$ and $\mathrm{Xe}^{2+}+\mathrm{Xe}$ collisions differ significantly from those reported for $\mathrm{e}^{-}+\mathrm{Xe}$ collisions $[19,21]$, in which the cross section maxima are observed much closer to the emission excitation threshold. From a purely classical perspective, the observation of higher heavy-particle emission excitation cross sections than those observed for electrons is surprising because the relative nuclear motion is substantially slower than the motion of the atomic electrons at all of the investigated energies, making momentum transfer between a heavy particle and an electron unlikely. The observed heavy-particle emission excitation cross sections must thus be the consequence of a nonclassical, nonadiabatic mechanism [32,33]. The observed increasing trend with energy is fully consistent with a nonadiabatic excitation mechanism.

In the case of the $\mathrm{Xe}^{2+}+\mathrm{Xe}$ system, the emission excitation cross sections reach a plateau near ion energies of $600 \mathrm{eV}(300 \mathrm{eV}$ per charge unit). For both charge states, charge transfer (two-electron charge transfer in the $\mathrm{Xe}^{2+}+\mathrm{Xe}$ system) is likely to play an important role in the excitation mechanism, and the excited atom may be associated with either the original ion or the target atom. This conclusion can be made because charge exchange occurs effectively at large interatomic distances in regions of weak interaction, whereas the electronic transitions leading to radiance occur at short range in regions of strong interaction.

Given the growing $\mathrm{Xe}^{+}$-induced emission cross sections with energy, it can be anticipated that the ionic contribution to the observed emission spectrum of a Xe-propelled HET grows with discharge voltage. Furthermore, the excitation rate scales with collision velocity, leading to an additional enhancement of ionic contributions with energy. As reported by Chiu et al. [19] and Karabadzhak et al. [20], a significant factor contributing to the sensitivity of the near-infrared spectrum to electron temperature in plasmas with low electron temperatures is the significant difference between the ion-induced and low-energy electron-induced nearinfrared spectra. With the higher ion-induced excitation rates at higher discharge voltages, the sensitivity of the diagnostic proposed by Karabadzhak et al. will be further enhanced. As the electron temperature is increased, the number of electrons with energies exceeding the excitation threshold increases, and the high-energy ion contribution becomes less significant.

By studying emissions simulated by the CRM of Karabadzhak et al. [20], which incorporates the present ion-collision excitation cross sections, we can quantify the contributions of ion-induced emission over a range of electron temperatures and ion energies, the latter of which approximately corresponds to HET discharge voltage.
In this CRM, $k_{\lambda_{e}}, k_{\lambda_{1}}$, and $k_{\lambda_{2}}$ are the rate coefficients for electron, singly charged ion, and doubly charged ion induced emissions, respectively, and the emission excitation rate for the line emitting at $\lambda$ obeys the relation

$$
\begin{aligned}
J_{\lambda} & \propto n_{0} n_{e}\left[k_{\lambda_{e}}\left(T_{e}\right)+\alpha_{1} k_{\lambda_{1}}\left(E_{1}\right)\right. \\
& \left.+\frac{1-\alpha_{1}}{2} k_{\lambda_{2}}\left(E_{2}\right)\right]\left[1+M\left(T_{e}, \alpha_{1}, \lambda\right)\right]
\end{aligned}
$$

Note that $k_{\lambda_{e}}$ is a function of the electron temperature, $k_{\lambda_{1}}$ and $k_{\lambda_{2}}$ are functions of the singly and doubly charged ion energies $E_{1}$ and $E_{2}$, and $M\left(T_{e}, \alpha_{1}, \lambda\right)$ is an intensity-enhancement factor that accounts for population of the $1 \mathrm{~s}_{5}$ metastable state through radiative transitions out of the $2 \mathrm{p}_{6-10}$ levels. Note that if we set $M, k_{\lambda_{1}}$, and $k_{\lambda_{2}}$ to zero, then Eq. (14) reduces to the coronal equilibrium model.

Because of inaccuracies in the emission excitation cross sections, as well as in some of the assumptions pertaining to metastable excitation, the CRM is considered to be more accurate if a spectrum of the full set of the near-infrared lines is simulated and fit to the observation through the variation of plasma parameters (i.e., electron temperature and ion charge ratio). However, for the purpose of clarity, we focus on the emission rates of only two lines, which allows us to discuss the ratio of the two rates rather than a best fit for all of the lines. Because Karabadzhak et al. [20] demonstrated the significant sensitivity of the $823 / 828$-nm intensity ratio to electron temperature, we shall use these lines. Furthermore, these two lines are attractive because of the spectral proximity that limits the effect of errors in the spectral-sensitivity calibration. Note that the $M$ factor must be retained in the following analysis because the 823-nm line is coupled to the $1 \mathrm{~s}_{5}$ metastable state. Therefore, additional cross sections measured in this work that are associated with other transitions into the $1 \mathrm{~s}_{5}$ state must be incorporated in the two-line analysis via the $M$ factor (see Karabadzhak et al. [20] for details on the $M$ factor).

Figure 4 shows the fraction of emission due to ion-impact excitation of the 823- and 828-nm lines as a function of electron temperature for the nine ion energies studied. HET plumes typically exhibit electron temperatures between $1-2 \mathrm{eV}$ in the far field (greater than $100 \mathrm{~mm}$ ) and approximately $10 \mathrm{eV}$ close to the exit plane of the thruster $[34,35]$. For xenon plasmas with $300-\mathrm{eV}$ ions, the contribution to the 823-nm line intensity by ion collisions is approximately $10 \%$ at an electron temperature of $5 \mathrm{eV}$ and rises rapidly with decreasing electron temperature. The contribution is particularly strong as the electron temperature approaches $2 \mathrm{eV}$. (Because of certain approximations, the CRM is inaccurate below $2 \mathrm{eV}$.) As the ion energy increases, so does the fraction of the emission produced by ion-impact processes. In fact, for the $900-\mathrm{eV}$ ions, the fraction of emission due to ion-impact excitation does not drop below $10 \%$ until the electron temperature is greater than $8.5 \mathrm{eV}$. This behavior is typical of all of the modeled lines involving transitions into the $1 \mathrm{~s}_{5}$ metastable state. The 828-nm line shows the lowest fraction of emission due to ion-induced excitation of all of the investigated lines. However, even here, the fraction is significant for electron temperatures between 2 and $4 \mathrm{eV}$, particularly for the higher 

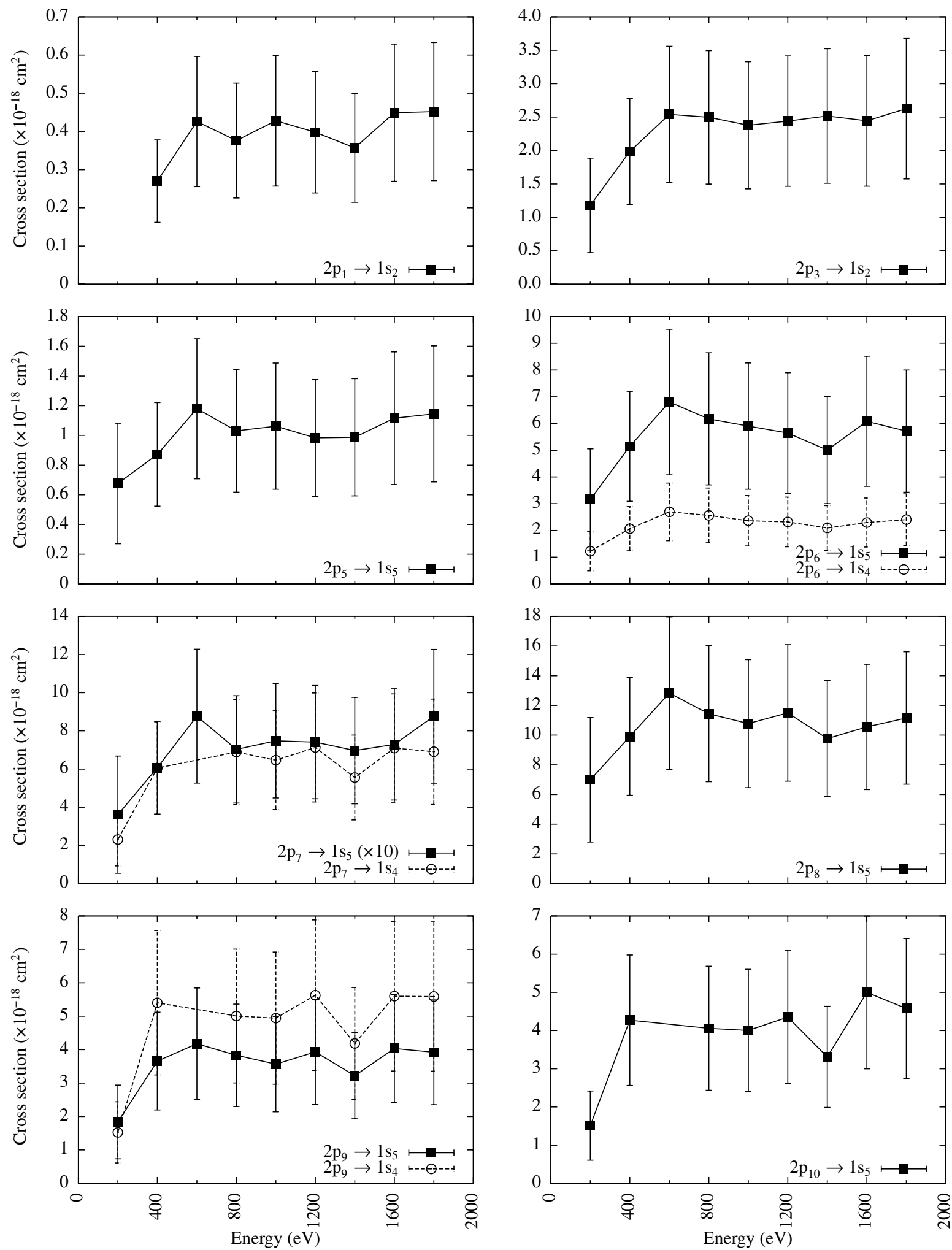

Fig. $3 \mathrm{Xe}^{2+}+\mathrm{Xe}$ emission excitation cross sections as a function of ion energy.

ion energies. Clearly, ion-induced emissions account for a significant portion of the luminescence on the xenon near-infrared lines in HET plumes.

Figure 5 shows the modeled electron temperature versus the ratio of the emission intensity of the 823-nm line to the intensity of the 828-nm line for four different ion energies. Given an experimentally measured intensity ratio, this two-line model can predict the electron temperature if the ion energy is known. Because of the high ioninduced emission cross sections, particularly for $\mathrm{Xe}^{+}$, failure to account for this excitation mechanism leads to increasingly erroneous results as the electron temperature is reduced or the ion energy is increased. This is further illustrated in Fig. $\underline{6}$, which compares the electron temperature that would be deduced from selected emission ratios using a coronalike CRM that neglects ion-induced excitation, with the results of the CRM incorporating the presently determined ion emission excitation cross sections. The failure to include ion-induced emission leads to increasingly erroneous results as the ion energy increases. These errors are 
Table $4 \mathrm{Xe}^{2+}+\mathrm{Xe}$ emission excitation cross sections $\left(10^{-18} \mathrm{~cm}^{2}\right)^{\mathrm{a}}$

\begin{tabular}{lccccccccccc}
\hline \hline Energy, eV & $789 \mathrm{~nm}$ & $823 \mathrm{~nm}$ & $828 \mathrm{~nm}$ & $835 \mathrm{~nm}$ & $841 \mathrm{~nm}$ & $882 \mathrm{~nm}$ & $895 \mathrm{~nm}$ & $905 \mathrm{~nm}$ & $916 \mathrm{~nm}$ & $980 \mathrm{~nm}$ & $992 \mathrm{~nm}$ \\
\hline 200 & - & $3.15^{\underline{b}}$ & $0.676^{\underline{b}}$ & $1.17 \underline{\underline{b}}$ & $0.360^{\mathrm{c}}$ & $6.99 \underline{\underline{b}}$ & $1.22^{\underline{b}}$ & $1.83^{\underline{b}}$ & $2.30^{\underline{\underline{b}}}$ & $1.51^{\underline{b}}$ & $1.52^{\underline{b}}$ \\
400 & 0.270 & 5.14 & 0.872 & 1.98 & 0.606 & 9.90 & 2.06 & 3.65 & 6.04 & 4.27 & 5.40 \\
600 & 0.425 & 6.80 & 1.17 & 2.54 & 0.877 & 12.8 & 2.69 & 4.17 & 7.88 & 6.75 & 6.26 \\
800 & 0.376 & 6.17 & 1.03 & 2.49 & 0.702 & 11.4 & 2.56 & 3.83 & 6.89 & 4.05 & 5.00 \\
1000 & 0.427 & 5.90 & 1.06 & 2.37 & 0.748 & 10.7 & 2.36 & 3.56 & 6.46 & 4.00 & 4.94 \\
1200 & 0.398 & 5.64 & 0.982 & 2.43 & 0.740 & 11.4 & 2.31 & 3.93 & 7.12 & 4.35 & 5.62 \\
1400 & 0.356 & 5.00 & 0.986 & 2.51 & 0.696 & 9.76 & 2.09 & 3.22 & 5.55 & 3.31 & 4.18 \\
1600 & 0.449 & 6.08 & 1.11 & 2.44 & 0.728 & 10.5 & 2.29 & 4.03 & 7.09 & 4.99 & 5.60 \\
1800 & 0.452 & 5.71 & 1.14 & 2.62 & 0.876 & 11.1 & 2.40 & 3.92 & 6.90 & 4.57 & 5.58 \\
\hline \hline
\end{tabular}

${ }^{a}$ All measurements have a $40 \%$ uncertainty unless otherwise marked b60\% uncertainty.

c85\% uncertainty.

particularly problematic at low electron temperatures, for which the relative deviation is largest.

For example, suppose a ratio of intensities in a certain location of the plume of an HET operating with a discharge voltage of $800 \mathrm{~V}$ is found to be $J_{823} / J_{828}=3.0$. This is a reasonable value, because Karabadzhak et al. [20] found ratios in HETs ranging between 1.1 and 4.5 from the discharge region to the plume of an anode-layer HET, and Matlock et al. [36] found ratios between $\sim 1$ and $\sim 8$ in the plume of a Busek BPT-200. If the coronalike model is used, an electron temperature of $4.4 \mathrm{eV}$ is determined. If, however, the full Karabadzhak et al. [20] CRM is used, an electron temperature $5.8 \mathrm{eV}$ is determined. The coronalike model underpredicts the electron temperature by about $25 \%$ in this case.

The sensitivity in the determination of the electron temperature from the ratio of two lines can be described by how rapidly that ratio changes with electron temperature:

$$
S\left(T_{e}, E_{i}\right) \equiv\left|\frac{\partial\left(J_{1} / J_{2}\right)}{\partial T_{e}}\right|_{T_{e}} \mid
$$
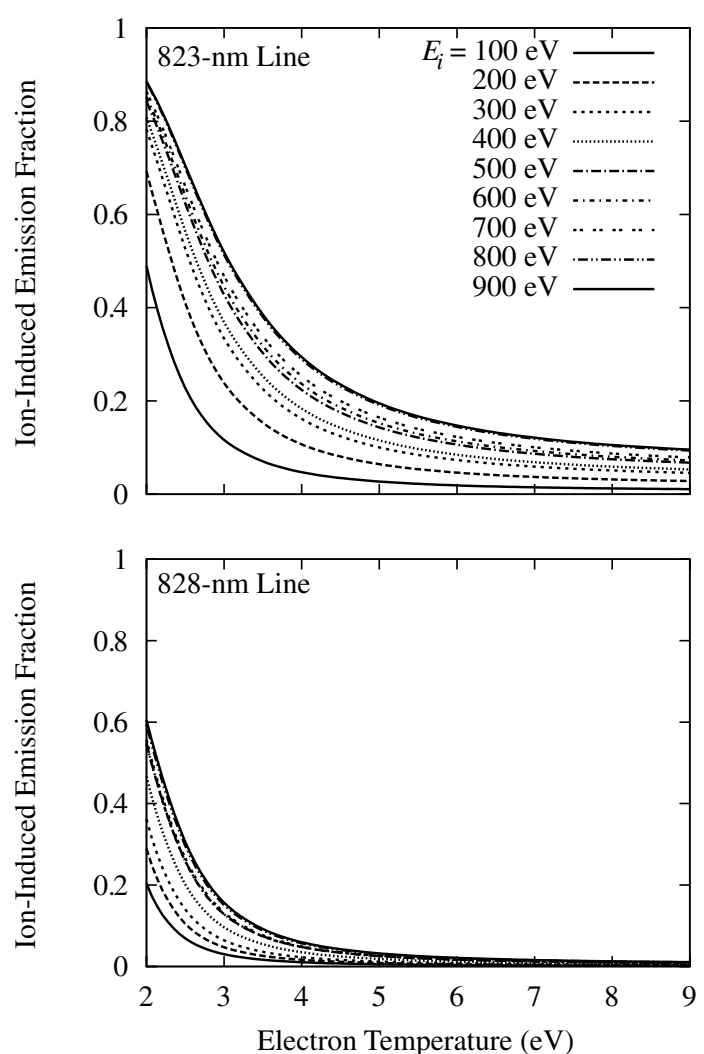

Fig. 4 Fraction of emission due to ion-induced excitation for the 823and 828-nm lines as a function of electron temperature for each of the ion energies measured in this work.
Because the ion and low-energy electron-induced near-infrared spectra are quite different, ion contributions add to the sensitivity of the CRM in determining the electron temperature. Figure 7 shows the sensitivity $823 / 828 \mathrm{~nm}$ emission ratio as a function of ion energy. For all temperatures, the sensitivity increases with ion energy. Particularly at low electron temperatures, the sensitivity of the $J_{823} / J_{828}$ ratio shows a dramatic increase as the ion energy is

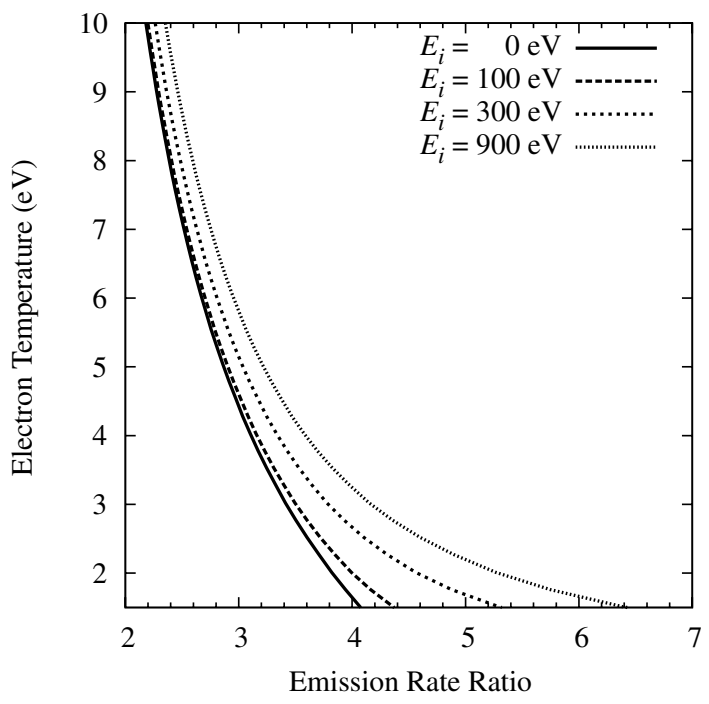

Fig. 5 Emission rate ratio $J_{823} / J_{828}$ as a function of electron temperature for select ion energies.

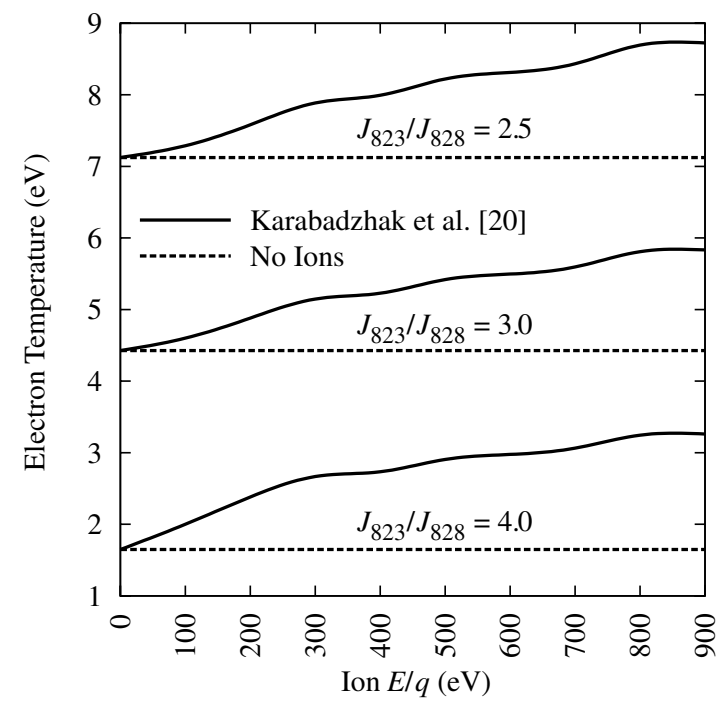

Fig. 6 Ion-energy dependence of derived electron temperature for select emission rate ratio $J_{823} / J_{828}$. 


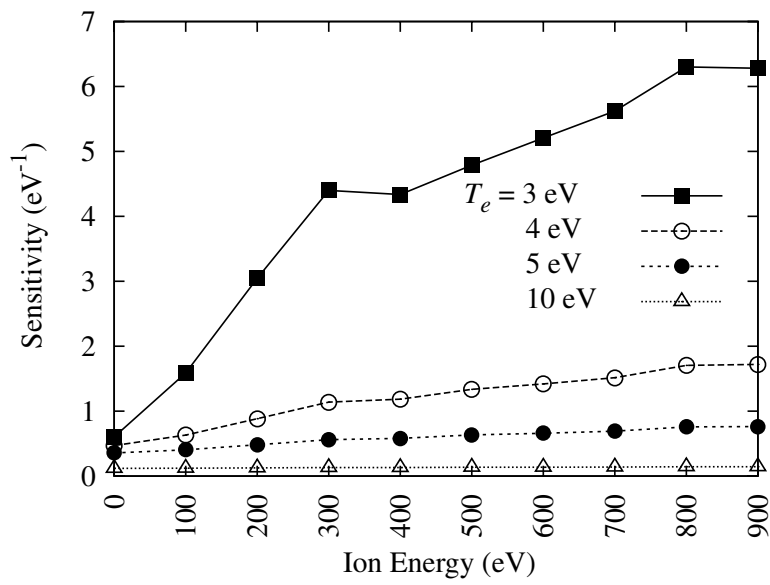

Fig. 7 Sensitivity of the 823- to 828-nm emission ratios to $T_{e}$ as a function of ion energy at select electron temperatures.

increased. It is fortuitous for electric propulsion research that the proper incorporation of ion-induced emissions into a CRM not only improves the model's accuracy in determining electron temperature, but also improves its sensitivity.

\section{Conclusions}

We report experimental emission excitation cross sections for 11 emission lines of neutral xenon impacted by $\mathrm{Xe}^{+}$and $\mathrm{Xe}^{2+}$ across an $E / q$ range from 100 to $900 \mathrm{eV}$. The cross sections are incorporated in a xenon plasma collisional-radiative model that includes emission excitation by energetic ions. Inclusion of an ion emission excitation mechanism is required to accurately model HET plasmas, in which ion-induced radiance may have comparable intensity with that produced by electron collisions, particularly at high ion energy and low electron temperature. Failure of a CRM to include the ioninduced emissions can result in errors in the determination of the electron temperature in the plume of an HET on the order of $25 \%$. The present cross sections extend the applicability of the Karabadzhak et al. [20] CRM to a broader range of HET operating voltages. The upgraded model can now also be applied to plasmas of low-specific-impulse gridded ion thrusters.

Additional measurements of ion-induced emission excitation cross sections for xenon would further extend the availability and usefulness of the Karabadzhak et al. [20] and other CRMs. In particular, the extension of the range of the $\mathrm{Xe}^{+}$- and $\mathrm{Xe}^{2+}$-induced cross sections to $2000 \mathrm{eV}$ per charge number would open these diagnostics to the bulk of gridded ion thrusters. Furthermore, measurements of the emission excitation cross sections of ionic lines through the processes 4 and 6 would improve the accuracy of the model at high electron temperatures and could also provide estimates of absolute electron, neutral, and ion densities.

\section{References}

[1] Jahn, R. G., Physics of Electric Propulsion, McGraw-Hill Series in Missile and Space Technology, McGraw-Hill, New York, 1968.

[2] Griem, H. R., Principles of Plasma Spectroscopy, Cambridge Monographs on Plasma Physics, Vol 2, Cambridge Univ. Press, Cambridge, England, U.K., 1997.

[3] Cooper, J., "Plasma Spectroscopy," Reports on Progress in Physics, Vol. 29, Pt. 1, 1966, pp. 35-130. doi:10.1088/0034-4885/29/1/302

[4] Selwyn, G. S., Optical Diagnostic Techniques for Plasma Processing, AVS Monograph Series, Vol. 11, American Vacuum Society Education Committee, New York, 1993.

[5] Fujimoto, T., "A Collisional-Radiative Model for Helium and Its Application to a Discharge Plasma," Journal of Quantitative Spectroscopy and Radiative Transfer, Vol. 21, No. 5, May 1979, pp. $439-455$. doi:10.1016/0022-4073(79)90004-9

[6] Malyshev, M. V. and Donnelly, V. M., "Trace Rare Gases Optical Emission Spectroscopy: Nonintrusive Method for Measuring Electron
Temperatures in Low-Pressure, Low-Temperature Plasmas," Physical Review E (Statistical Physics, Plasmas, Fluids, and Related Interdisciplinary Topics), Vol. 60, No. 5, Nov. 1999, pp. 6016-6029. doi:10.1103/PhysRevE.60.6016

[7] Manzella, D. H., "Stationary Plasma Thruster Plume Emissions," 23rd International Electric Propulsion Conference, Electric Rocket Propulsion Society Paper 93-097, September 13-16 1993.

[8] Karabadzhak, G. F., Semenkin, A. V., and Manzella, D. H., "Investigation of TAL Optical Emissions," 25th International Electric Propulsion Conference, Electric Rocket Propulsion Society Paper 97 131, Aug. 1997.

[9] Leray, P., Bonnet, J., and Pigache, D., "Spacially Resolved Emission Spectroscopy Along an SPT Channel: Interpretation of Data by a Collisional-Radiative Model," 25th International Electric Propulsion Conference, Electric Rocket Propulsion Society Paper 97-054, Aug. 1997.

[10] Meezan, N. B., Hargus, W. A., Schmidt, D. P., and Cappelli, M. A., "Optical Study of Anomalous Electron Transport in a Laboratory Hall Thruster," 35th AIAA/ASME/SAE/ASEE Joint Propulsion Conference, Los Angeles, CA, AIAA Paper 99-2284, June 1999.

[11] Darnon, F., Garrigues, L., Boeuf, J. P., Bouchoule, A., and Lyszyk, M., "Spontaneous Oscillations in a Hall Thruster," IEEE Transactions on Plasma Science, Vol. 27, No. 1, Feb. 1999, pp. 98-99. doi:10.1109/27.763063

[12] Komurasaki, K., Sakurai, Y., and Kusamoto, D., "Optical Oscillations in a Hall Thruster," 34th AIAA/ASME/SAE/ASEE Joint Propulsion Conference, Cleveland, OH, AIAA Paper 98-3051, July 1998.

[13] Bouchoule, A., Philippe-Kadlec, C., Prioul, M., Darnon, F., Lyszyk, M., Mangne, L., Pagnon, D., Roche, S., Touzeau, M., Béchu, S., Lasgorceix, P., Sadeghi, N., Dorval, N., Marque, J.-P., and Bonnet, J., "Transient Phenomena in Closed Electron Drift Plasma Thrusters: Insights Obtained in a French Cooperative Program," Plasma Sources Science and Technology, Vol. 10, No. 2, May 2001, pp. 364 377. doi:10.1088/0963-0252/10/2/326

[14] Pagnon, D., Touzeau, M., and Lasgorceix, P., "Control of the Ceramic Erosion by Optical Emission Spectroscopy: Parametric Studies of SPT100-ML," 40th AIAA/ASME/SAE/ASEE Joint Propulsion Conference, Fort Lauderdale, FL, AIAA Paper 2004-3773, July 2004.

[15] Karabadzhak, G. F., Semenkin, A. V., and Tverdokhlebov, S. O., "Evaluation of a Xenon Operating Hall Thruster Body Erosion Rate Through Analysis of Its Optical Spectra," 37th AIAA/ASME/SAE/ ASEE Joint Propulsion Conference, Salt Lake City, UT, AIAA Paper 2001-3889, July 2001.

[16] Gulczinski, F. S., and Gallimore, A. D., "Near-field Ion Energy and Species Measurements of a 5-kW Hall Thruster," Journal of Propulsion and Power, Vol. 17, No. 2, Apr. 2001, pp. 418-427.

[17] King, L. B., and Gallimore, A. D., "Mass Spectral Measurements in the Plume of an SPT-100 Hall Thruster," Journal of Propulsion and Power, Vol. 16, No. 6, Nov. 2000, pp. 1086-1092.

[18] Lochte-Holtgreven, W., "Evaluation of Plasma Parameters," Plasma Diagnostics, edited by W. Lochte-Holtgreven, North-Holland, Amsterdam, 1968, pp. 135-213.

[19] Chiu, Y.-H., Austin, B. L., Williams, S., and Dressler, R. A., "Passive Optical Diagnostic of Xe Propelled Hall Thrusters, Part 1: Emission Cross Sections," Journal of Applied Physics, Vol. 99, June 2006, Paper 113304 doi:10.1063/1.2195018

[20] Karabadzhak, G. F., Chiu, Y.-H., and Dressler, R. A., "Passive Optical Diagnostic of Xe Propelled Hall Thrusters, Part 2: CollisionalRadiative Model," Journal of Applied Physics, Vol. 99, June 2006, Paper 113305 doi:10.1063/1.2195019

[21] Fons, J. T., and Lin, C. C., "Measurement of the Cross Sections for Electron-Impact Excitation into the 5p $\mathrm{p}^{5} 6 \mathrm{p}$ Levels of Xenon," Physical Review A, Vol. 58, No. 6, Dec. 1998, pp. 4603-4615. doi:10.1103/PhysRevA.58.4603

[22] Manzella, D. H., and Jacobson, D., "Investigation of Low-Voltage/ High-Thrust Hall Thruster Operation," 39th AIAA/ASME/SAE/ASEE Joint Propulsion Conference, Huntsville, AL, AIAA Paper 2003-5004, July 2003.

[23] Butler, G. W., Yuen, J. L., Tverdokhlebov, S. O., Semenkin, A. V., and Jankovsky, R. S., "Multimode, High Specific Impulse Hall Thruster Technology," 36th AIAA/ASME/SAE/ASEE Joint Propulsion Conference, Huntsville, AL, AIAA Paper 2000-3254, July 2000.

[24] Pote, B., and Tedrake, T., "Performance of a High Specific Impulse Hall Thruster," 27th International Electric Propulsion Conference, Electric Rocket Propulsion Society Paper 01-35, Oct. 2001.

[25] Hofer, R. R., Jankovsky, R. S., and Gallimore, A. D., "High-Specific 
Impulse Hall Thrusters, Part 1: Influence of Current Density and Magnetic Field," Journal of Propulsion and Power, Vol. 22, No. 4, July 2006, pp. 721-731. doi: $10.2514 / 1.15952$

[26] Hofer, R. R., Jankovsky, R. S., and Gallimore, A. D., "High-Specific Impulse Hall Thrusters, Part 2: Efficiency Analysis," Journal of Propulsion and Power, Vol. 22, No. 4, July 2006, pp. 732-740. doi:10.2514/1.15954

[27] Belikov, M. B., Gorshkov, O. A., and Rizakhanov, R. N., "Investigation of a Hall Thruster of $1.5 \mathrm{~kW}$ Class of Power at Specific Impulses Up to 3000 s," 36th AIAA/ASME/SAE/ASEE Joint Propulsion Conference, Huntsville, AL, AIAA Paper 2000-3253, July 2000.

[28] Patterson, M., Grisnik, S. P., and Soulas, G. C., "Scaling of Ion Thrusters to Low Power," 25th International Electric Propulsion Conference, Electric Rocket Propulsion Society Paper 97-098, Aug. 1997.

[29] Soulas, G. C., Foster, J. E., and Patterson, M., "Performance of Titanium Optics on a NASA $30 \mathrm{~cm}$ Ion Thruster," 36th AIAA/ASME/ SAE/ASEE Joint Propulsion Conference, Huntsville, AL, AIAA Paper 2000-3814, July 2000.

[30] Radzig, A. A., and Smirnov, A. A., Reference Data on Atoms, Molecules, and Ions, Springer-Verlag, New York, 1985.

[31] Aymar, M., and Coulombe, M., "Theoretical Transition Probabilities and Lifetimes in Kr I and Xe I Spectra," Atomic Data and Nuclear Data
Tables, Vol. 21, No. 6, June 1978, pp. 537-566.

doi:10.1016/0092-640X(78)90007-4

[32] Bransden, B. H., and McDowell, M. R. C., Charge Exchange and the Theory of Ion-Atom Collisions, Oxford Univ. Press, New York, 1992.

[33] Delos, J. B., "Theory of Electronic Transitions in Slow Atomic Collisions," Reviews of Modern Physics, Vol. 53, No. 2, Apr. 1981, pp. 287-357. doi:10.1103/RevModPhys.53.287

[34] Beal, B. E., Gallimore, A. D., Haas, J. M., and Hargus, W. A., "Plasma Properties in the Plume of a Hall Thruster Cluster," Journal of Propulsion and Power, Vol. 20, No. 6, Nov. 2004, pp. 985-991. doi:10.2514/1.3765

[35] Haas, J. M., "Low-Perturbation Interrogation of the Internal and Nearfield Plasma Structure of a Hall Thruster Using a High-speed Probe Positioning System,” M.S. Thesis, Univ. of Michigan, Ann Arbor, MI, 2001.

[36] Matlock, T. S., Larson, C. W., Hargus, W. A., and Nakles, M. R., “An Inversion Method for Reconstructing Hall Thruster Plume Parameters from the Line Integrated Measurements," 43rd AIAA/ASME/SAE/ ASEE Joint Propulsion Conference, Cincinnati, OH, AIAA Paper 2007 5303, July 2007.

R. Myers Associate Editor 